\title{
Visibility of an afterimage in the presence of a second afterimage*
}

\author{
JANETTE ATKINSON \\ The Psychological Laboratory, University of Cambridge, Cambridge, England
}

Visibility of an afterimage has been measured for a single rectangular bar on its own and in the presence of a second bar. Visibility of each bar is increased if the two bars are in the same orientation and closer than $1 \mathrm{deg}$ (this effect being called "facilitation") and is decreased if the bars are in different orientations (this effect being called "inhibition"). These interactions also depend on the relative size of the bars. If a pattern of two bars is viewed dichoptically, rivalry between the bars occurs causing a mutual decrease in visibility. The rivalry effect and the orientation specific effects discussed above are additive in the dichoptic viewing condition. The orientation-specific effects have been likened to those found in other psychophysical studies of angle detection.

The nonuniform fading of patterned afterimages was first observed by Helmholtz (1866; see 1925 ed.). Quantitative studies of the fragmentation of afterimages have more recently been made by Evans (1967), Evans and Wells (1967), Mackinnon, Forde, and Piggins (1969), and Atkinson (1970, 1972). The conclusion from all of these studies is that structured fragmentation is a result of activity in contour-detecting units within the visual system.

Pritchard, Heron, and Hebb (1960) studied the fragmentation of an optically stabilized image, using a contact lens device, and found that a figure in one part of the stimulus field could modify the visibility of neighboring parts of the stimulus. However, they did not quantify this Gestalt type of "field effect."

The present experiment was carried out to see whether one afterimage could affect the visibility of another nearby afterimage. The visibility of an afterimage was measured with and without a second afterimage. Only the visibility of the entire image was timed, no record was made of fragmentary appearances. Viewing conditions, orientation, size, and separation of the two afterimages were varied in different experiments.

\section{METHOD}

\section{Apparatus and Procedure}

The apparatus and procedure were essentially the same as that described in detail in a previous paper (Atkinson,

\footnotetext{
* Janette Atkinson was awarded the George Henry Lewes studentship from the Physiological Laboratory, University of Cambridge, and the Henry George Plimmer fellowship from the Imperial College of Science and Technology, University of London, to finance this research. The author would like to thank Dr. F. W. Campbell, Dr. L. A. Rirss, and Dr. O. J. Braddick for their helpful criticisms of this paper.
}

1972). The afterimages were produced by a Braun electronic flashgun, with flash duration of approximately 4 msec and light output of $160 \mathrm{~J}$. Patterns were produced by cutouts in a black mask which was placed immediately in front of the flash.

For monocular viewing, $S$ fixated a spot attached to the mask with his right eye, his left eye being covered throughout the trial. For dichoptic

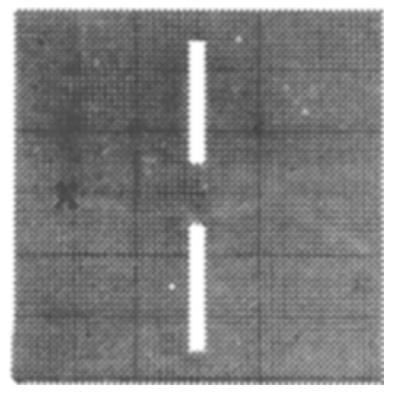

viewing, two black screens were positioned so that the right eye saw only one bar in the pattern and the left eye saw only the second bar in the pattern. The S's eyes were horizontally aligned with the fixation spot $28.5 \mathrm{~cm}$ from it. In the dichoptic viewing condition, $S$ triggered the flashgun himself, so that his eyes were accurately lined up at the moment of the flash and the afterimage that followed had the same relative positioning properties as the stimulus pattern. Any $S$ who had great difficulty in aligning the pattern accurately in the practice trials was eliminated from the experiments. Immediately after the flash, $\mathbf{S}$ closed his eyes and kept them closed throughout the trial time, thus maintaining a constant background luminance for viewing the afterimages.

The trial time was always $60 \mathrm{sec}$ immediately following the flash. S always had $15 \mathrm{~min}$ adaptation before the first trial and $5 \mathrm{~min}$ adaptation between trials. The $S$ was asked not to blink, to keep his head still, and to make as few voluntary eye movements as possible.

Small silent two-way switches were used by $S$ to record visibility of the
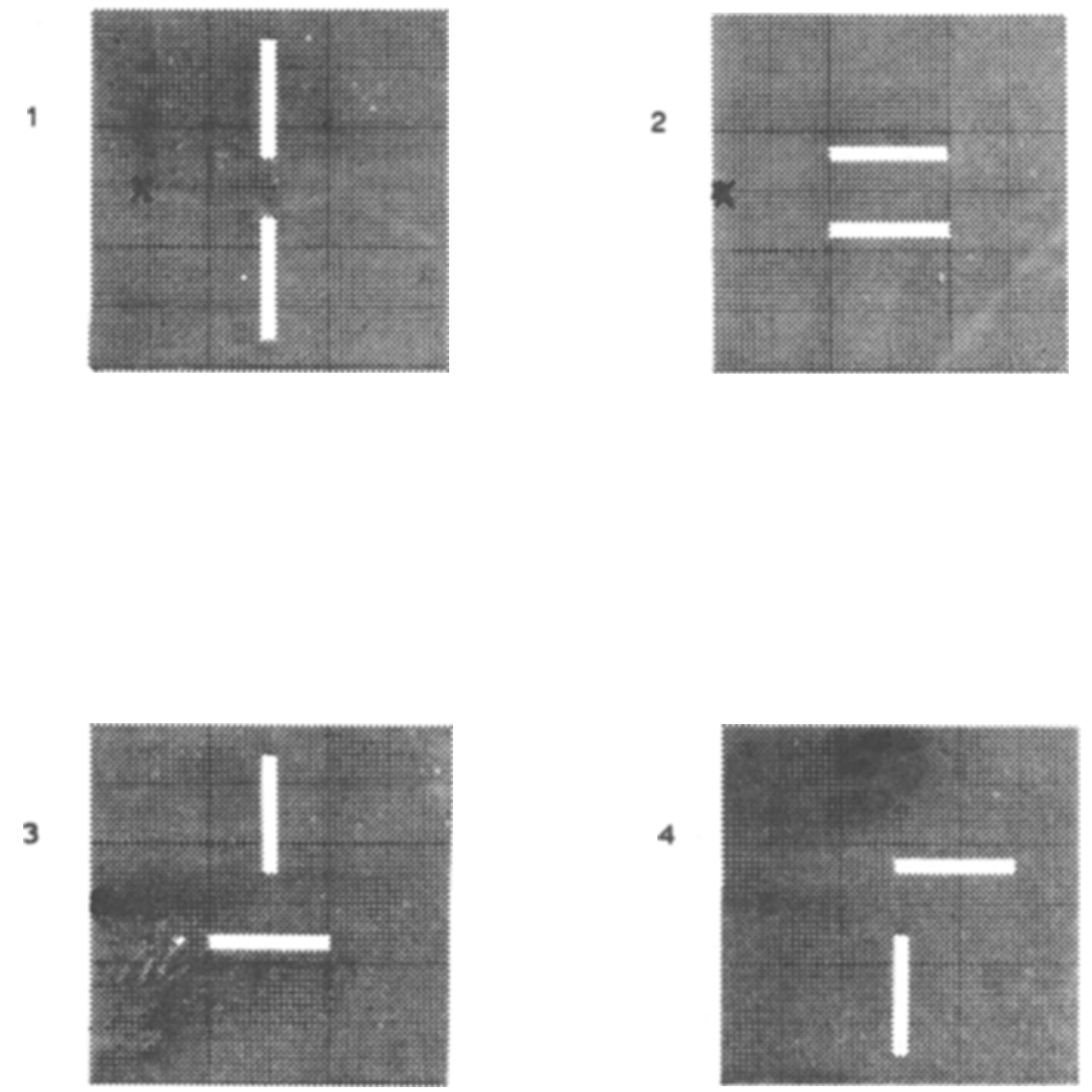

Fig. 1. The four patterns used in Experiment 1. $X$ marks the fixation spot. Patterns 1 and 2 were also used in Experiment 2. 


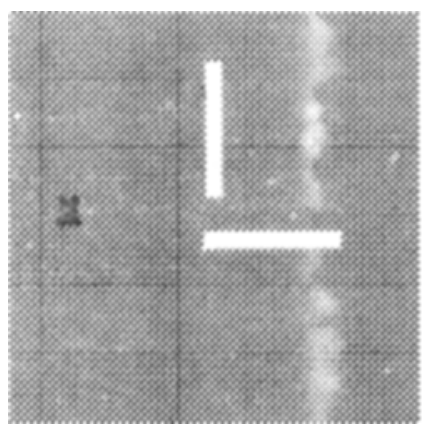

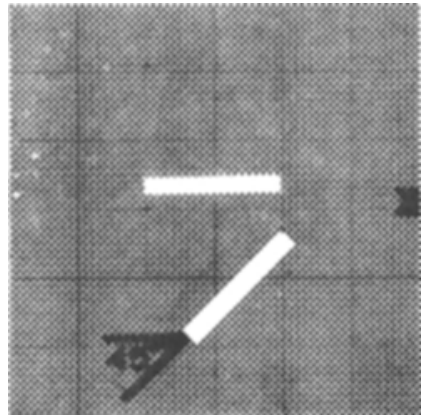

3

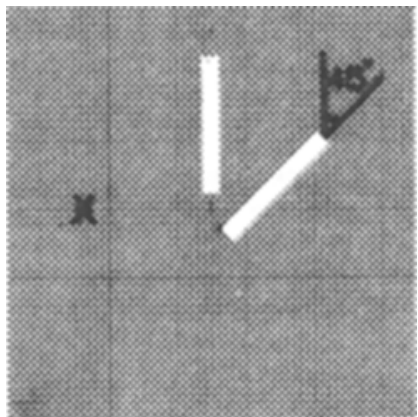

Fig. 2. The three patterns used in Experiment 3. $\times$ marks the fixation spot.

afterimage. The $\mathbf{S}$ depressed a switch when a particular afterimage was visible and released the switch when the image disappeared. Visibility was measured in seconds of trial time for which the afterimage was visible, as recorded on counters connected to the switches. No knowledge of results was given during testing.

Orders of presentation were used which balanced for practice effects between different stimuli and viewing conditions.

\section{Subjects}

All Ss were students at the University of Cambridge. They were between 17 and 35 years of age and were naive regarding the nature of the experiments. All had normal or corrected-to-normal vision.

\section{Experiment 1}

In this experiment and in subsequent experiments involving the same patterns, the term "a pattern of two vertical bars" will mean a pattern in which the two bars are aligned so that one bar is directly above the other (as in Fig. 1, Pattern 1). "A pattern of two horizontal bars" will mean two parallel bars horizontally oriented, one above the other (as in Fig. 1, Pattern 2). It is obvious that these two patterns do not have the same configurational properties, nor do they cover the same area of visual field.

The two additional patterns used in Experiment 1 are shown in Fig. 1. In all patterns, the separation of the bars
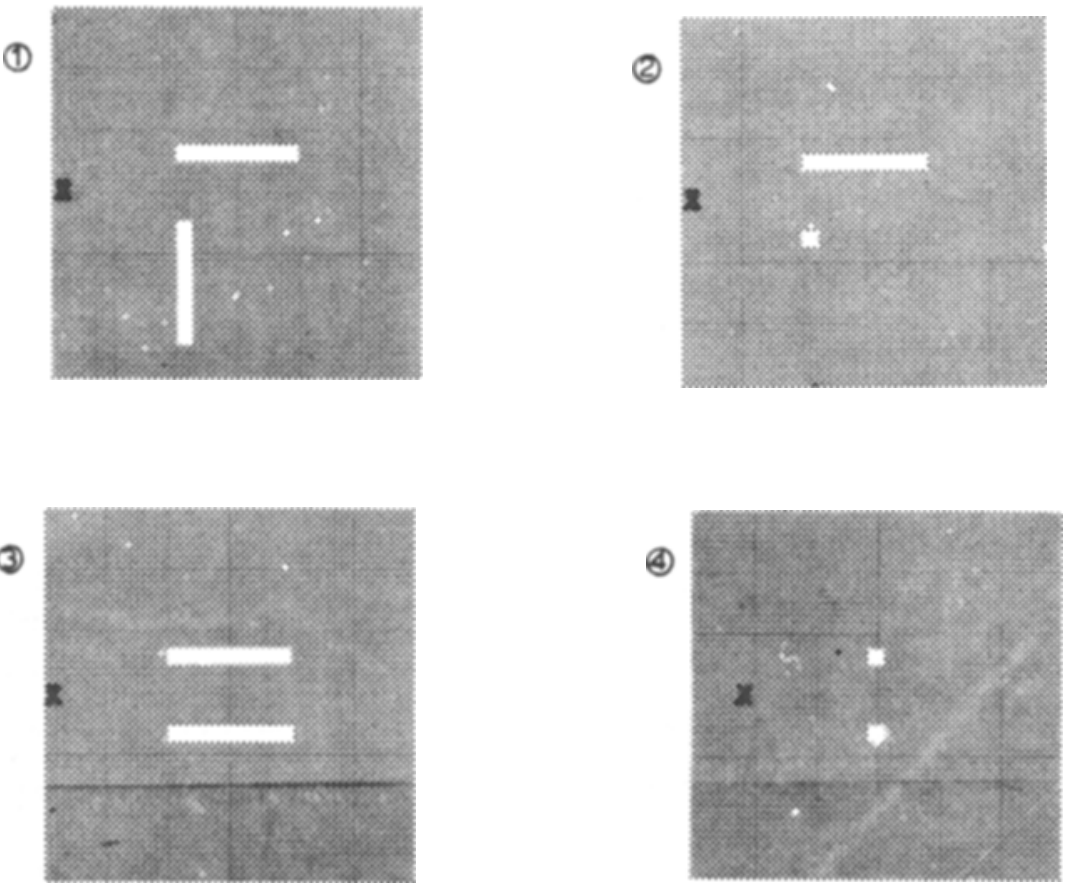

Fig. 3. The four patterns used in Experiment $4 . \times$ marks the fixation spot.

was constant at 1 deg of visual angle. The fixation point was always $2 \mathrm{deg}$ from the bars, as indicated in Fig. 1.

For all patterns, both monocular and dichoptic viewing conditions were used. In the dichoptic condition, S's right eye viewed the top bar only and the left eye the bottom bar only.

For each of the patterns, two comparisons were made: (1) Between the visibility of the top bar when viewed on its own (the bottom bar being concealed for that trial) and the visibility of the same bar when both bars were present. (2) Between the visibility of the bottom bar when viewed on its own and the visibility of the same bar when both bars were present.

Thus, the visibility of an afterimage was compared with and without a second afterimage. Single vertical images were compared only with other vertical images at the same retinal locus, and horizontal images were compared with horizontal images.

Eleven is recorded the total visibility for each of the bars in each pattern. Each $\mathbf{S}$ had five trials on each condition, the first being a practice.

\section{Experiment 2}

Patterns 1 and 2 from Fig. 1 were used again in Experiment 2 . The distance separating the bars was varied, being $0.5,1,2$, or 3 deg. Each bar was viewed alone and with the second bar, making four experimental conditions in all for each separation. Of course, separation is meaningless for single bar patterns, but each single bar pattern acted as a control at the same eccentricity as the bars of the relevant two-bar pattern.

Eleven Ss completed five trials on each condition, the first trial in each condition being a practice. For each pattern, the mean visibility for the top and bottom bars were calculated.

\section{Experiment 3}

The patterns used are shown in Fig. 2 . 
The total visibility was measured for each bar with and without a second bar. Three different separations were used, these being $0.5,1$, and 2 deg. Each of the five Ss had three trials on each condition, the first being a practice.

\section{Experiment 4}

In Experiments 1-3, all the bars were 2 deg in length; in Experiment 4, the relative sizes of the bars were varied as shown in Fig. 3. Monocular and dichoptic viewing conditions were used for each patterm. Fifteen Ss completed three trials on each condition, the first being a practice. The visibility of each bar was measured for the bar on its own and accompanied by the second bar in each pattern.

\section{RESULTS}

Experiment 1

A preliminary analysis was carried out to determine whether the requirement to respond to two separate afterimages (in the same trial time), rather than one, introduced any bias into the results which would mask any potential differences between patterns. Trials given in Experiment 1, in which two bars were in the stimulus pattem, were split into two types. In Type 1, the $\mathbf{S}$ had only to record the visibility of one bar, although a second bar was present. In Type 2, the visibilities of two afterimages were recorded simultaneously by the $S$, using two switches (one in each hand). The results for Type 1 and Type 2 were compared, using analysis of variance, and no significant difference was found between the two $[F(1,110)$ $=0.3, p>.05]$. This meant that the requirement to respond to two afterimages rather than one was not affecting the amount of visibility recorded. The results for the two types of trial were therefore pooled for further analysis.

Analysis of variance on the results for mean visibilities in Experiment 1 showed that: (1) There was a significant difference between monocular and dichoptic viewing conditions; visibility was always higher for monocular viewing $[F(1,10)=$ $70.3, p<.01]$. (2) There was no significant difference between the total visibilities of vertical and horizontal bars $[F(1,10)=2.9$, $p>.05]$ for monocular or dichoptic viewing. (3) Visibility was altered by the presence and orientation of another afterimare in the stimulus pattern $[F(3,30)=52.1, p<.01]$. The mean visibility for each bar, with and without a second bar, is shown in Fig. 4 for each pattern.

Monocular viewing. The difference between the visibility for a vertical bar

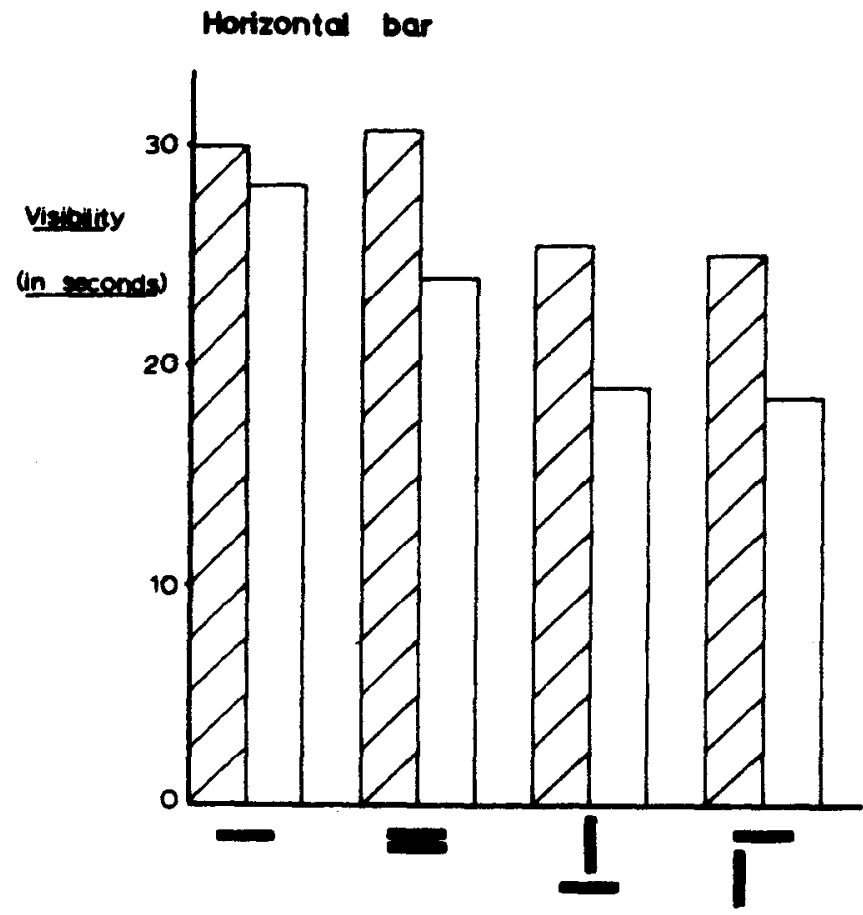

Vertical bar

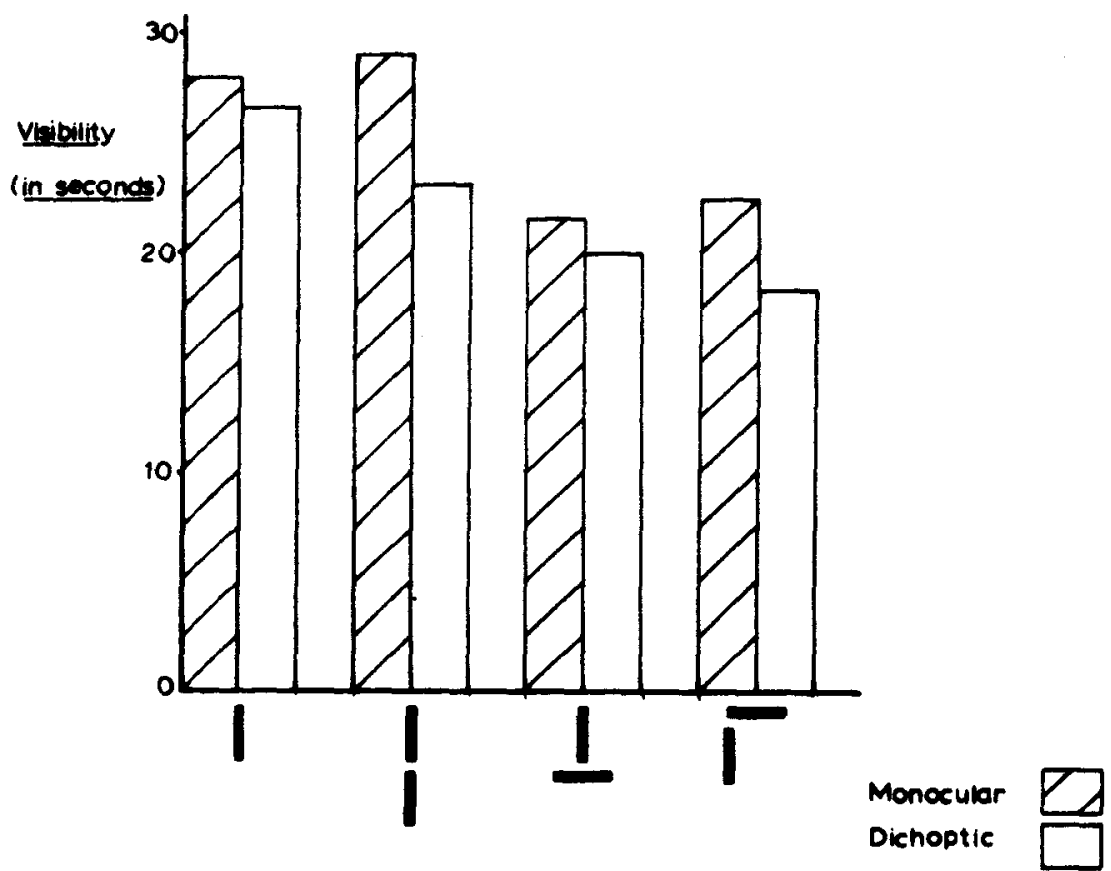

Fig. 4. Visibility (in seconds) for a horizontal bar alone and accompanied by another horizontal bar or by a vertical bar (top graph). Visibility (in seconds) for a vertical bar alone and accompanied by another vertical bar or by a horizontal bar (bottom graph).

alone compared with a vertical bar's visibility when accompanied by a horizontal bar (see Fig. 4) was significantly different on the Newman-Keuls and Tukey (a) tests $(p<.01)$. This lowering of visibility 
Table 1

\begin{tabular}{|c|c|c|c|c|c|c|c|c|c|c|c|c|}
\hline & \multicolumn{6}{|c|}{ One Horizontal Bar } & \multicolumn{6}{|c|}{ One Vertical Bar } \\
\hline & \multicolumn{2}{|c|}{ Pattem 2} & \multicolumn{2}{|c|}{ Pattern 3} & \multicolumn{2}{|c|}{ Pattern 4} & \multicolumn{2}{|c|}{ Pattern 1} & \multicolumn{2}{|c|}{ Pattern 3} & \multicolumn{2}{|c|}{ Pattern 4} \\
\hline & $\mathbf{M V}$ & $\mathbf{F}$ & $\mathbf{M V}$ & $\mathbf{I}$ & $\mathbf{M V}$ & $\mathbf{I}$ & $\mathbf{M V}$ & $\mathbf{F}$ & $\mathbf{M V}$ & $\mathbf{I}$ & $\mathbf{M V}$ & $\mathbf{I}$ \\
\hline $\begin{array}{l}\text { Mean Visibility } \pm \\
\text { Inhlbition/Facilitation }\end{array}$ & \multicolumn{2}{|c|}{$23.7-1.0$} & \multicolumn{2}{|c|}{$19.0+5.0$} & \multicolumn{2}{|c|}{$19.3+5.0$} & \multicolumn{2}{|c|}{$25.1-1.0$} & 17.8 & $\mathbf{5 . 0}$ & \multicolumn{2}{|c|}{$18.3+5.0$} \\
\hline Total (in Sec) & \multicolumn{2}{|c|}{22.7} & \multicolumn{2}{|c|}{24.0} & \multicolumn{2}{|c|}{24.3} & \multicolumn{2}{|c|}{24.1} & \multicolumn{2}{|c|}{22.8} & \multicolumn{2}{|c|}{23.3} \\
\hline
\end{tabular}
called "inhibition." The term is merely used as a description and is not meant to imply anything about underlying mechanisms. The same effect was found for the visibility of a horizontal bar accompanied by a vertical bar.

Visibility was raised when a vertical bar was accompanied by a second vertical bar, although this effect was small and did not reach significance on the Newman-Keuls or Tukey (a) tests. The same was true for patterns of two horizontal bars. This small increase in visibility produced by the presence of a second parallel bar will be referred to as "facilitation." by a differently oriented bar will be

These monocular effects of "facilitation" and "inhibition" are dependent on relative orientation of the bars and can therefore be said to be "orientation specific."

Dichoptic viewing results. For all four patterns, visibility was lower for dichoptic than for monocular viewing, as shown in Fig. 4. For dichoptic viewing, the drop in visibility caused by the presence of a second afterimage could be due to either a type of binocular rivalry that is dependent on the relative orientation of the bars, or to rivalry between bars (which is equal in amount for any pattern) plus the monocular orientation-specific effect

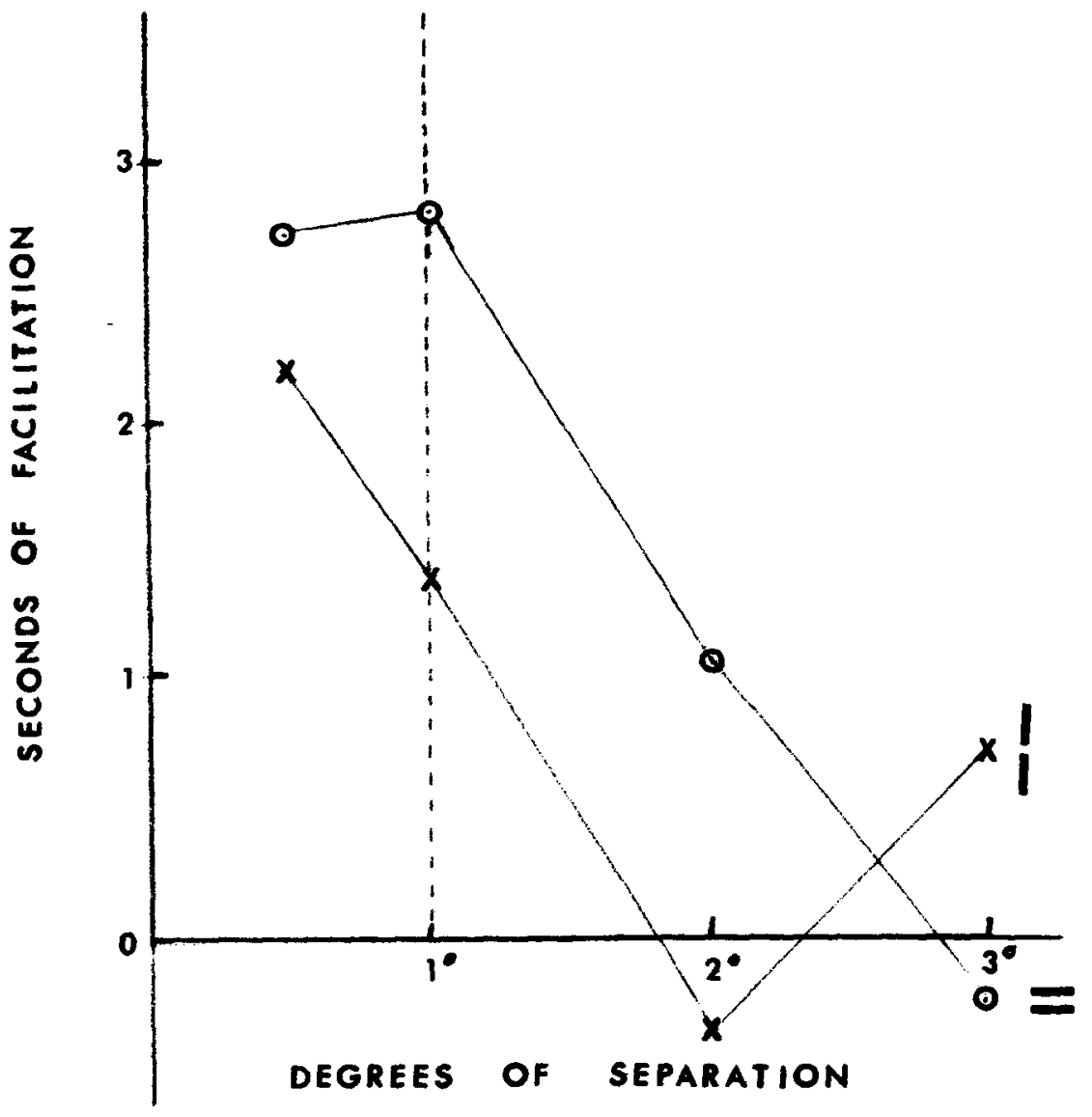

Fig. 5. Facilitation (i.e., the amount of increase, in seconds, of visibility due to the presence of a second bar in the same orientation) as a function of separation of the bars (in degrees). The dotted line shows the separation up to which facilitation is a significant effect. discussed above. The second alternative assumes that the two factors (orientation and rivalry) are additive.

If the first hypothesis is correct, then the interactive factor for Viewing Condition by Pattern would be significant. This interactive factor was not significant at the .01 level, but was at the .05 level. As the .01 level of $p$ has been used for all experimental results in this study, this particular result will be considered nonsignificant, and, as such, the result is support for the hypothesis that the orientation-specific effect is transferred in dichoptic viewing and is s u p r i posed on the nonorientationspecific rivalry effect.

From the monocular results, the orientation-specific effect was found to be approximately $5 \mathrm{sec}$ of inhibition for a pattern of two bars at a right angle to one another and $1 \mathrm{sec}$ of facilitation for two bars that are in the same orientation. If these amounts for facilitation and inhibition are subtracted from the dichoptic visibility for each bar, the calculated visibility for each bar without the orientation-specific effects is much the same for all patterns (approximately $24 \mathrm{sec}$ visibility per bar). These calculations are shown in Table 1. From this calculation, a value of approximately $4 \mathrm{sec}$ reduction in visibility is found to be due to rivalry which is not orientation specific.

\section{Experiment 2}

The effect of one bar on the visibility of another was calculated for each bar at each separation by subtracting the mean visibility of a bar on its own from the mean visibility of the same bar in the presence of the second bar. Only patterns of two vertical or two horizontal bars were used in this experiment. Facilitation, at each separation, is shown in Fig. 5 . Facilitation occurred when either two vertical or two horizontal bars were close together, but not when they were more than $1 \mathrm{deg}$ apart. A small but statistically reliable facilitation was found using analysis of variance $[F(1,10)=10.9, p<.01]$. On further comparison of the means for various separations, using the Newman-Keuls and Tukey (a) tests, the facilitation was significant for only the two smaller separations of the bars ( 0.5 and $1 \mathrm{deg}$ ). 
Experiment 3

As in the previous two experiments, the visibility was calculated for each bar with and without a second bar; the difference between these two visibilities is shown in Fig. 6. The amount of inhibition varied with the separation of the bars, being highest when the bars were $1 \mathrm{deg}$ apart $[F(2,8)=63.1, p<.01]$. This was true for both the right angle and oblique bar patterns. The extent of inhibition did not vary significantly from pattern to pattern, i.e., there was no greater inhibition between bars at right angles to one another than between bars at $45 \mathrm{deg}$ to one another $[F(5,20)=0.9, p>.05]$.

\section{Experiment 4}

The inhibition and facilitation were calculated for each pattern (see Fig. 3 for patterns used) and are plotted in Fig. 7. From analysis of variance, significant differences were found (1) between monocular and dichoptic viewing conditions $[F(1,14)=38.1$, $p<.01]$ and (2) between visibilities of a bar with and without a second bar $[F(6,84)=11.1, p<.01]$.

For dichoptic viewing, all visibilities were found to be shifted approximately 4 sec in the inhibitory direction, although the absolute amount of visibility varied from pattern to pattern. From the analysis of variance, there was no significant interaction between viewing conditions and the effects $[F(6,84)=$ $2.9, p>.05]$. This result confirms the result of Experiment 1 and shows that the two factors (rivalry and orientation-specific effect) are separate and additive.

The amount of inhibition or

\section{Intibition}
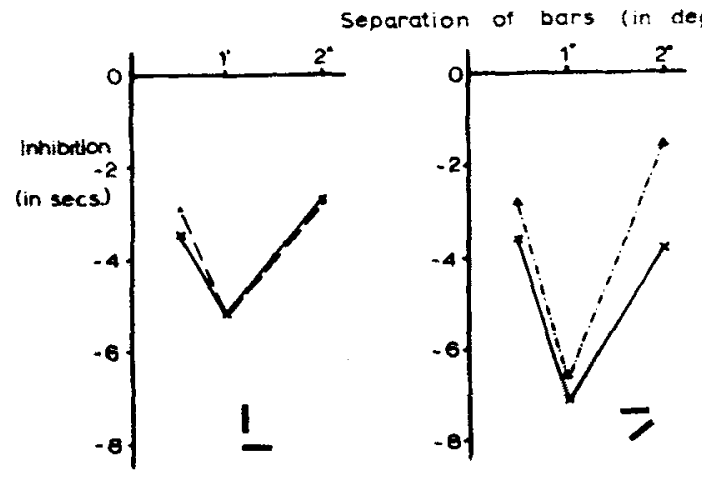

Fig. 6. Inhibition (i.e., the amount of decrease, in seconds, of visibility of a bar, due to the presence of a second bar in a different orientation) as a function of separation of the bars (in degrees).
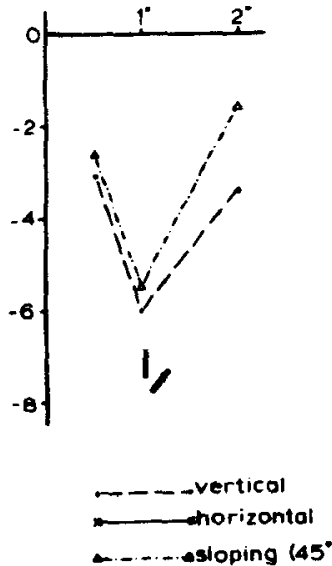

\section{Facilitation and Inhibition}

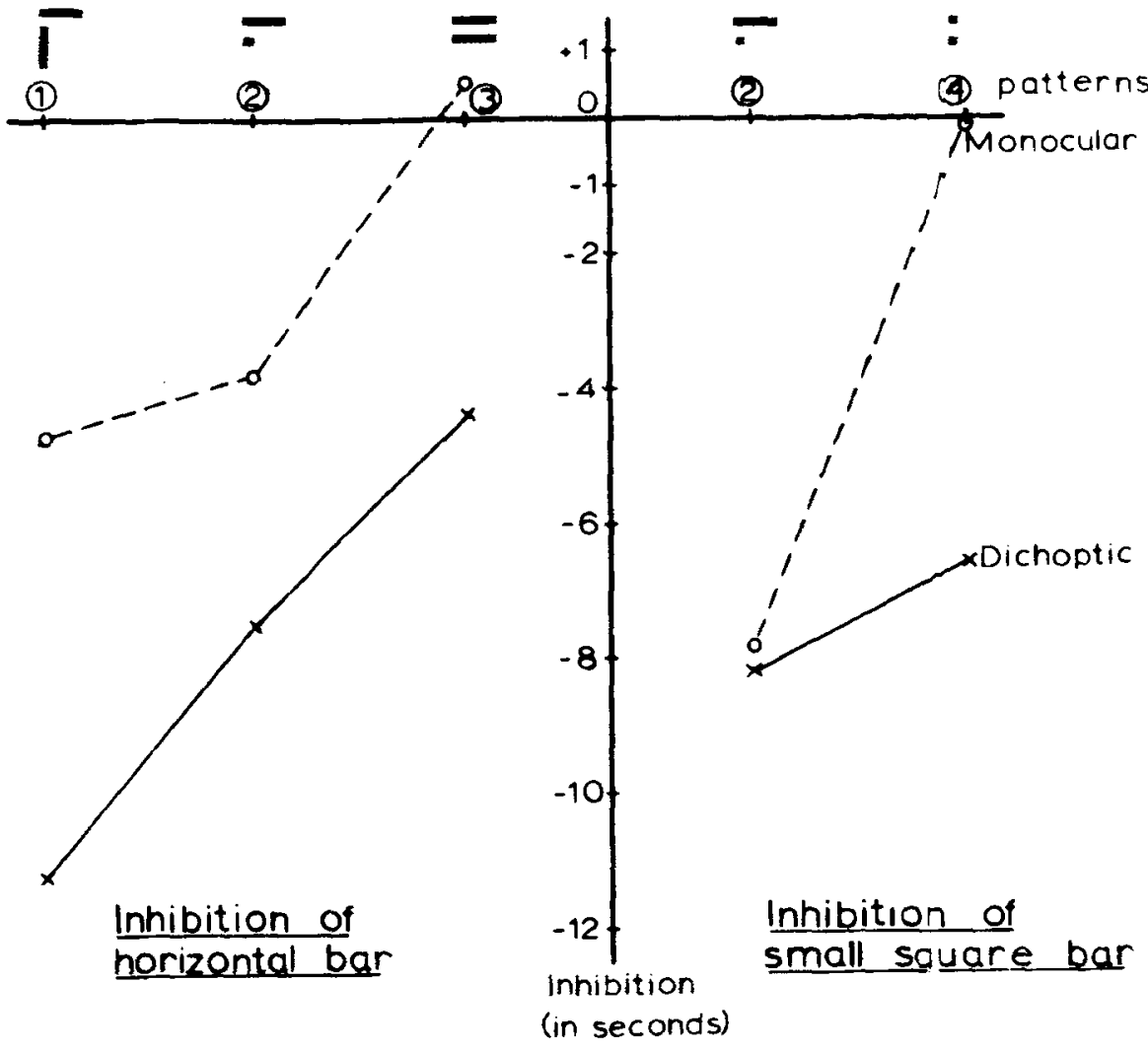

Fig. 7. Orientation specific effects (facilitation and inhibition) for different bars in the four patterns of Experiment 4.

facilitation depended upon the relative size of the bars in each pattern. The small square, as seen in Fig. 3, Pattern 2, was inhibited by the horizontal bar but was not inhibited

by another small square (Fig. 3, Pattern 4). However, this result could be explained merely in terms of the difference in orientation that was present in Fig. 3, Pattern 2, but not present in Fig. 3, Pattern 4. In Fig. 3, Patterns 1 and 2 , the orientation of the two bars was at $90 \mathrm{deg}$, but in Pattern 1, the bars were also the same size. Inhibition of the horizontal bar by the small square smaller than inhibition of the horizontal bar by the vertical bar of the same size. Thus, it is seen that the relative size, as well as the relative orientation, has an appreciable effect on the amount of inhibition.

\section{DISCUSSION}

Facilitatory and inhibitory effects of one afterimage or another have been demonstrated. These effects depend on the relative orientation, size, and spacing of the two images. For two identical images in the same orientation, as close or closer than $1 \mathrm{deg}$, there is a small facilitatory effect. For two bars in different orientations, there is a large mutual inhibition (approximately $5 \mathrm{sec}$ inhibition). The results of Experiment 3 showed a higher 
inhibition at 1 -deg separation than at 0.5- or 2-deg separations.

These results can be accounted for if it is postulated that for any two-bar pattern there is a small facilitation of visibility if the bars are close together, and superimposed on this effect there is an orientation-specific inhibition which operates only if the bars are in different orientations. Both effects decrease in magnitude as the spacing of the bars is increased, the facilitatory effect declining at a greater rate than the inhibitory effect. Now, at small separations ( $0.5 \mathrm{deg})$, both facilitatory and inhibitory effects will be operating in opposition to one another (for any pattern where the bars are in different orientations); at 1-deg separation, both effects have declined but are still operating and the facilitatory effect is much weaker than it was at $0.5-\mathrm{deg}$ separation, so that the final value for inhibition appears higher at 1 than at $0.5 \mathrm{deg}$. At a separation of $2 \mathrm{deg}$, there is no facilitation but the inhibitory factor is still operating; because of the increase in separation, the inhibition is weaker at 2- than at 1-deg separation.

A previous study by Atkinson (1970) showed that the unitary visibility of two bars at a right angle with no gap between their ends was the same as the unitary visibility of two bars in the same orientation joined end to end (contour length being equal for both patterns). It is obvious, therefore, that the orientation-specific inhibition demonstrated in the above experiments does not operate unless there is a space between the ends of the bars. The size of this space, necessary to cause inhibition, may be related to the distance over which perceptual "closure" of the space is possible. Several informal observations made on two experienced Ss revealed that there was a definite minimum size of gap at which the bars inhibited one another, and if the gap was smaller than this critical size, "closure" was often observed. The minimum size of gap was approximately $0.25 \mathrm{deg}$. It seems likely that the minimum gap size relates in some way to the size of overlap of receptive fields of the units in the visual system responsible for the perceptual phenomena, but it would seem presumptuous to try to specify the exact mechanism any further at this point.

Bl akemore, Carpenter, and Georgeson (1970) and Bouma and
Andriessen (1970) have reported studies in which a change in perceived orientation of a line has been brought about by a second line at an angle to the first. This orientation-specific effect might be similar to the effect reported in the present study on afterimages; the orientation-sensitive units that are operating in both these experimental situations laterally inhibit one another if they are tuned to different, but proximal, orientations. There is as yet no electrophysiological evidence to provide a basis for such inhibition between cortical units.

The inhibitory effects found for dichoptic viewing have been explained in terms of a combination of the monocular orientation-specific effects (reported in the preceding section) and a rivalry effect which is independent of relative orientation. Binocular rivalry between two optically stabilized images has been reported by Krauskopf and Riggs (1959) and Ditchburn and Pritchard (1960). Kaufman (1963) and Levelt (1965), using nonstabilized images, also found that rivalry depended on the relative positioning of the two images. However, none of these studies compared dichoptic results with monocular results in the same way as in the present afterimage study, and so it is impossible to compare the possible effect of orientation differences across studies.

Cohen (1961), using a stabilized vertical line as stimulus to one eye, found that a similar nonstabilized line to the other eye increased the visibility of the stabilized line. His results are contrary to what would be expected from the present result on afterimages, which would predict a decrease in visibility on presentation of any other contour as a nearby stimulus to the other eye.

In conclusion, the results of Experiments 1-4 show that afterimages mutually interact, causing changes in their total visibilities. The nature of these interactions depends on the relative orientation, size, and spacing of the two images and also on the viewing condition used. A type of binocular rivalry which is constant for all patterns is superimposed upon orientation-specific effects, producing lower visibilities in the dichoptic viewing condition than in the monocular.

The orientation-specific effect for afterimages has been likened to that found in other psychophysical studies on angle estimation. Thus, the notion of in h i b i ion betwee $n$ contour-detecting units, which has been put forward to account for the latter results, may also be applicable to the interactions of afterimages.

\section{REFERENCES}

ATKINSON, J. A study of perceptual analysis using stabilized images. Unpublished doctoral dissertation, University of Cambridge, 1970.

ATKINSON, J. The effect of size, retinal locus, and orientation on the visibility of a single afterimage. Perception \& Psychophysics, 1972, 12 (2B), 213-217. BL AKEMORE, C., CARPENTER, R. H. S. \& GEORGESON, M. A. Lateral inhibition between orientation detectors in the human visual system. Nature, 1970, 228 , 37-39.

BOUMA, H., \& ANDRIESSEN, J. J Induced changes in the perceived orientation of line segments. Vision Research, 1970, 10, 333-349.

COHEN, H. B. The effect of contralateral stimulation on visibility with stabilized retinal images, Canadian Journal of Psy chology, 1961, 15, 212-219.

DITCHBURN, R. M.. \& PRITCHARD, R W. Binocular vision with two stabilized retinal images. Quarterly Journal of Experimental Psychology, 1960, 12, 26-65.

EVANS, C, R. Further studies of pattern perception and a stabilized retinal image: The use of prolonged afterimages to achieve perfect stabilization. British Journal of Psychology, 1967, 58 315-327.

EVANS, C. R. \& WELLS, A. M Fragmentation phenomena associated with binocular stabilization. British Journal of Physiological Optics, 1967, 24, 45-50.

HELMHOLTZ, H. von. Handbuch der physiologischen Optik. Vol. II. (Trans. J. C. Southall). Optical Society of America 1925. P. 231.

KAUFMAN, L. On the spread of suppression and binocular rivalry. Vision Research, 1963, 3, 401-415.

KRAUSKOPF, J., \& RIGGS, L. A. Interocular transfer in the disappearance of stabilized images. American Journal of Psychology, 1959, 72, 248-252.

LEVELT, W. J. M. On binocular rivalry Soesterberg, The Netherlands: National Defence Research Organization, 1965.

MacKINNON, G. E.. FORDE, J., \& PIGGINS, D. J. Stabilized images steadily fixated figures and prolonged afterimages. Canadian Journal of Psy chology, 1969, 23, 184-195.

DITCHBURN, R. W. \& PRITCHARD, R M. Binocular vision with two stabilized retinal images. Quarterly Journal of Experimental Psychology, 1960, 12 , 26-32.

PRITCHARD, R. M., HERON, W., \& HEBB. D. $O$. Visual perception approached by the method of stabilized images. Canadian Journal of Psychology, 1960, 14, 67-77.

(Accepted for publication June 9, 1972.) 